\title{
New Ascomycete Record for Turkish Mycobiota
}

\section{Türk Mikobiyotası için Yeni Askomiset Kaydı}

\section{Research Article}

\section{Ömer F. Çolak ${ }^{1 *}$ and Oğuzhan Kaygusuz ${ }^{2}$}

'Süleyman Demirel University, Vocational School of Health Services, East Campus, Isparta, Turkey. 2Pamukkale University, Faculty of Science and Arts, Department of Biology, Kınıklı, Denizli, Turkey.

\section{A B S TR ACT}

n the current study, Peziza succosella (Le Gal \& Romagn.) M.M. Moser ex Aviz.-Hersh. \& Nemlich was reported for the first time from Turkey. Microscopic drawings and description of the taxon were given together with morphological photograph. In addition the current list of the genus Peziza in Turkey was presented.

\section{Key Words}

Biodiversity, Peziza, New record, Turkish mycobiota.

\section{öz}

u çalışmada, Peziza succosella (Le Gal \& Romagn.) M.M. Moser ex Aviz.-Hersh. \& Nemlich Türkiye'den ilk kez rapor edildi. Taksonun tanımlanması ve mikroskopik çizimleri morfolojik fotoğrafı ile birlikte verildi. Buna ek olarak, Türkiye'deki Peziza cinsinin güncel listesi sunuldu.

\section{Anahtar Kelimeler}

Biyoçeşitlilik, Peziza, Yeni kayıt, Türk mikobiyotası. 


\section{Introduction}

iological diversity or biodiversity, which as concept is product of the last 30 years, is "the variety of life", and refers collectively to variation at all levels of biological organization. Investigation of this topic is the most important task of taxonomists [1-3].

Many studies have been performed on macrofungal biodiversity by Turkish mycologists, and approximately 2400 taxa have been reported. Among this number, the Peziza Dill. ex Fr., which is the largest genus of the family Pezizaceae Dumort with over 100 species in the world, is represented with 27 species in Turkey until now [4-6].

The purpose of this study was to contribute to the biodiversity of this country by reporting a new interesting record of the genus Peziza.

\section{MATERIALS and METHODS}

The specimens were collected from Söke (Aydın) in 2014. Morphological and ecological characteristics of the samples were noted and photographed in their natural habitats. After field studies, specimens were taken to the laboratory. Microscopic characters were observed by light microscope using Melzer's reagent and distilled water. The identification of species was carried out using the literature [7-9]. The collections were deposited at the personal fungarium in Süleyman Demirel University.

\section{RESULTS}

A short description of Peziza succosella was given below. The systematic of taxon is in accordance with Index Fungorum [10].

Fungi Bartling

Ascomycota Whittaker

Pezizomycotina O.E. Erikss. \& Winka

Pezizomycetes O.E. Erikss. \& Winka

Pezizomycetidae Locq.

Pezizales J. Schröt.

Pezizaceae Dumort.

Peziza Dill. ex Fr.

Peziza succosella (Le Gal \& Romagn.) M.M. Moser ex Aviz.-Hersh. \& Nemlich (Figure 1)

Syn: Galactinia succosella Le Gal \& Romagn.

Hymenium smooth, greyish brown; apothecia 10-20 mm broad, initially disc shaped, slightly irregular at older stage, fragile, sessile to sub-sessile; outer surface smooth-glabrous, pale grey. Asci 250-350 × 15-17 $\mu \mathrm{m}$, cylindrical shaped, operculate, unitunicate, amyloid, 8-spored, uniseriate. Ascospores 15-19 $\times$ 8-11 $\mu \mathrm{m}$, ellipsoid, usually uniguttulate, ornamented (when examined with in Melzer's reagent), irregular warts (approx. 1.5 $\mu \mathrm{m})$ with partially reticulate surface. Paraphyses, the same length as asci and one third wide, with septa and extended apex. Habitat: in small groups, on the damp ground of the woods, in spring [7-9].

Specimen Examined: Turkey, Aydın province, Söke district, Doğanbey village around, altitude 5 meters, on soil, Quercus spp. and Pinus spp. mxed forest, 18.01.2014 (ÖFÇ 657).

\section{DISCUSSION}

Peziza succosella is identified as a new record for Turkish mycobiota. According to recent studies, twenty seven Peziza species have been reported in Turkey to the present date $[5,6,10-12]$. In the present study, Peziza succosella is reported as 28th member of Turkish Peziza (Table 1).

Other species that have been reported as Peziza spp. but now synonyms; $P$. ampelina Pass. (=Pyrenopeziza ampelina (Pass.) Rehm) [56,57], P. amphora Quél. (=Helvella solitaria P. Karst.) [33], P. celtica (Boud.) M.M. Moser (=Pachyella celtica (Boud.) Häffner) [15], P. coccinea Jacq. (=Sarcoscypha coccinea (Gray) Boud.) [5,58], P. erucaeformis Batsch (incorrect) [5].

Peziza succosa differs from the $P$. succosella by its yellowish olivaceous-brown hymenium. Also $P$. succosa size of apothecia is bigger than $P$. succosella. In these two species, apothecia when injured immediately exuding a juice which quickly turns yellow. On the contrary, juice of $P$. michelii becomes yellowish after a while. Also its hymenium initially violet, then brownish $[7,8,59,60]$. 

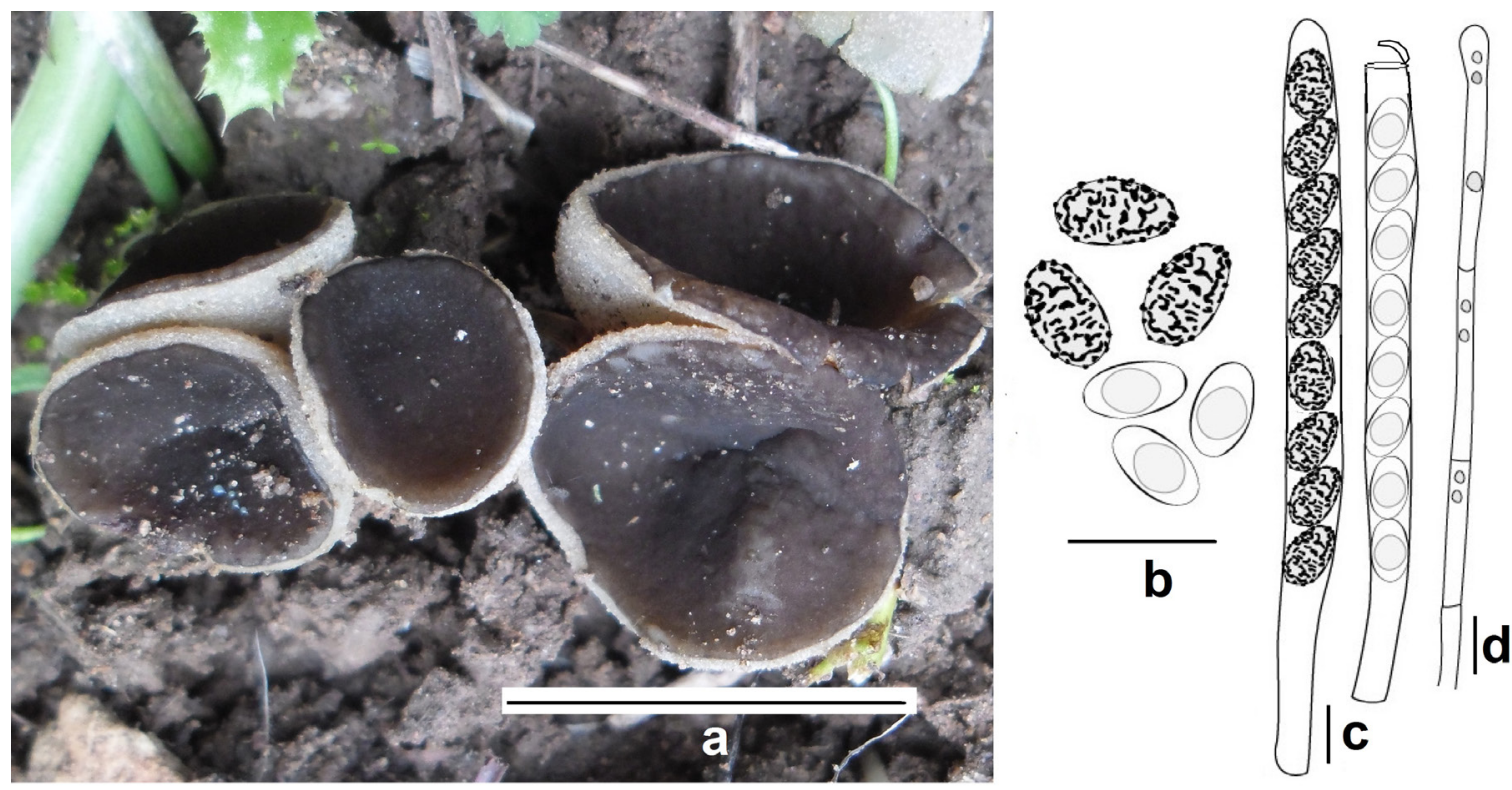

Figure 1. Peziza succosella, a). ascocarp, b). ascospores, c). ascus and d). paraphyse, (scale bars: $a=20 \mathrm{~mm} ; \mathrm{b}, \mathrm{c}$ and d= 20 $\mu \mathrm{m}$ ) (photo by Ö.F. ÇOLAK, line drawings by O. KAYGUSUZ).

Table 1. List of the genus Peziza in Turkey.

\begin{tabular}{|c|c|c|c|c|}
\hline Peziza species & $\begin{array}{c}\text { Solak et al. } 2007 \\
\text { [58] }\end{array}$ & $\begin{array}{c}\text { Sesli and Denchev } \\
2008 \text { [5] }\end{array}$ & Solak et al. 2015 [6] & References \\
\hline $\begin{array}{c}\text { P. ammophila Durieu } \\
\text { \& Lév. }\end{array}$ & $(-)$ & $(+)$ & $(+)$ & [13] \\
\hline P. arenaria Osbeck & $(-)$ & $(+)$ & $(+)$ & [14] \\
\hline $\begin{array}{c}\text { P. arvernensis Roze } \\
\text { \& Boud. }\end{array}$ & $(+)$ & $(+)$ & $(+)$ & [15-19] \\
\hline P. badia Pers. & $(+)$ & $(+)$ & $(+)$ & [20-22] \\
\hline P. cerea Sowerby & $(+)$ & $(+)$ & $(+)$ & [23] \\
\hline $\begin{array}{l}\text { P. depressa Pers. } \\
\text { (Syn: P. applanata } \\
\text { (Hedw.:Fr.) Alb. \& } \\
\text { Schwein) }\end{array}$ & $(+)$ & $(+)$ & $(+)$ & {$[18,24-27]$} \\
\hline P. domiciliana Cooke & $(+)$ & $(+)$ & $(+)$ & {$[15,18,28,29]$} \\
\hline $\begin{array}{c}\text { P. echinospora P. } \\
\text { Karst. }\end{array}$ & $(-)$ & $(-)$ & $(+)$ & {$[30]$} \\
\hline $\begin{array}{c}\text { P. fimeti (Fuckel) E.C. } \\
\text { Hansen }\end{array}$ & $(-)$ & $(+)$ & $(-)$ & [31] \\
\hline $\begin{array}{l}\text { P. granularis } \\
\text { Donadini }\end{array}$ & $(-)$ & $(-)$ & $(-)$ & [12] \\
\hline $\begin{array}{l}\text { P. granulosa } \\
\text { Schumach. }\end{array}$ & $(+)$ & $(+)$ & $(+)$ & {$[28,32,33]$} \\
\hline $\begin{array}{c}\text { P. Iobulata (Velen.) } \\
\text { Svr ek }\end{array}$ & $(-)$ & $(-)$ & $(+)$ & {$[34]$} \\
\hline
\end{tabular}




\begin{tabular}{|c|c|c|c|c|}
\hline $\begin{array}{l}\text { P. michelii (Boud.) } \\
\text { Dennis }\end{array}$ & $(+)$ & $(+)$ & $(+)$ & [32] \\
\hline P. micropus Pers. & $(+)$ & $(+)$ & $(+)$ & {$[16-35]$} \\
\hline $\begin{array}{c}\text { P. moravecii (Svr ek) } \\
\text { Donadini }\end{array}$ & $(-)$ & $(+)$ & $(+)$ & {$[36]$} \\
\hline $\begin{array}{c}\text { P. phyllogena } \\
\text { Cooke (Syn: P. } \\
\text { badioconfusa Korf) }\end{array}$ & $(-)$ & $(-)$ & $(-)$ & {$[37-41]$} \\
\hline P. praetervisa Bres. & $(-)$ & $(-)$ & $(+)$ & [29] \\
\hline $\begin{array}{c}\text { P. pseudoviolacea } \\
\text { Donadini }\end{array}$ & $(-)$ & $(-)$ & $(-)$ & [11] \\
\hline $\begin{array}{l}\text { P. punctispora } \\
\text { (Pfister) Donadini }\end{array}$ & $(-)$ & $(-)$ & $(+)$ & {$[42]$} \\
\hline P. repanda Pers. & $(+)$ & $(+)$ & $(+)$ & {$[20,22,40,43]$} \\
\hline $\begin{array}{l}\text { P. ripensis E.C. } \\
\text { Hansen }\end{array}$ & $(-)$ & $(-)$ & $(-)$ & [11] \\
\hline P. saniosa Schrad. & $(-)$ & $(+)$ & $(+)$ & [44] \\
\hline P. sepiatra Cooke & $(-)$ & $(-)$ & $(+)$ & [45] \\
\hline P. succosa Berk. & $(+)$ & $(+)$ & $(+)$ & {$[18,19,29,46]$} \\
\hline P. succosella & $(-)$ & $(-)$ & $(-)$ & This study \\
\hline $\begin{array}{l}\text { P. varia (Hedw.) Alb. } \\
\text { \& Schwein. }\end{array}$ & $(+)$ & $(+)$ & $(+)$ & {$[29,41,47]$} \\
\hline P. vesiculosa Bull. & $(+)$ & $(+)$ & $(+)$ & {$[28,48-52]$} \\
\hline P. violacea Pers. & $(+)$ & $(+)$ & $(+)$ & [53-55] \\
\hline
\end{tabular}

\section{References}

1. C. Lévêque, J.C. Mounolou, Biodiversité-2e éd.: Dynamique biologique et conservation, Dunod, (2008) 248p.

2. H.H. Başıbüyük, A. Yılmaz, S. Kılınç (eds), Biyoçeşitlilik, Biyolojik Devinimler ve Koruma, Palme yayıncılık, Ankara, (2013) 248p.

3. K.J. Gaston, J.I. Spicer, Biodiversity: an introduction, John Wiley \& Sons, (2013) 208p.

4. P.F. Kirk, P.F. Cannon, D.W. Minter, J.A. Stalpers, Dictionary of the fungi, 10th ed., CAB International, Wallingford, UK, (2008) 771p.

5. E. Sesli, C.M. Denchev, Checklists of the myxomycetes, larger ascomycetes, and larger basidiomycetes in Turkey, Mycotaxon, 106 (2008) 65-67. + [complete version, 1-145, new version uploaded in January 2014].

6. M.H. Solak, M. Işıloğlu, E. Kalmış, H. Allı, Macrofungi of Turkey, Checklist (Vol. 2), Üniversiteliler Ofset, İzmir, (2015) 280p.

7. L. Hansen, H. Knudsen (eds), Nordic macromycetes: Ascomycetes (Vol. 1), Nordsvamp, Copenhagen, (2000) 285p.
8. G. Medardi, Atlante fotografico degli Ascomicet d'Italia, Fondazione Centro Studi Micologici dell'Associazione Micologica Bresadola, Trento, (2006) 454p.

9. S. Jabeen, T. Ashraf, A.N. Khalid, Peziza succosella and its ectomycorrhiza associated with Cedrus deodara from Himalayan moist temperate forests of Pakistan, Mycotaxon, 130 (2015) 455-64.

10. http://www.indexfungorum.org/names/names.asp (2017).

11. A. Kaya, Contributions to the macrofungal diversity of Atatürk Dam Lake basin, Turk. J. Bot., 39 (2015) 16272.

12. i. Acar, Y. Uzun, Peziza granularis Donadini Türkiye mikobiyotası için yeni bir kayıt, Yüzüncü Yıl Üniv. Fen Bil. Ens. Dergisi, 21 (2016) 39-42.

13. I. Akata, A.E. Yaprak, A new Peziza record for Turkish mycobiota, BioDiCon, 6 (2013) 32-34.

14. A. Kaya, Macrofungal diversity of Adıyaman province (Turkey), Mycotaxon, 110 (2009) 43-46.

15. G. Kaşık, H.H. Doğan, C. Öztürk, S. Aktaş, Türkiye mikoflorası için Ascomycetes' ten yeni kayıtlar, Selçuk Üniv. Fen-Ed. Fak. Fen Dergisi, 20 (2002) 75-81.

16. A. Türkoğlu, Macrofungal diversity of Babadağ (Denizli, Turkey), Afr. J. Biotechnol., 7 (2008) 192-200. 
17. Z. Kırış, M.G. Halıcı, I. Akata, H. Allı, Macrofungi of Akdağmadeni (Yozgat/Turkey) and Gemerek (Sivas/ Turkey), BioDiCon, 5 (2012) 53-58.

18. I. Akata, Y. Uzun, A. Kaya, Macromycetes determined in Yomra (Trabzon) district, Turk. J. Bot., 38 (2014) 999-1012.

19. i. Türkekul, H. Işık, Contribution to the macrofungal diversity of Yozgat Province (Turkey), Mycotaxon, 131 (2006) 483-510.

20. M. Öner, A Contribution to the knowledge of common Turkish higher fungi, Mycopathologia et Mycologia Applicate, 47 (1972) 369-373.

21. M. Öner, T. Gezer, A contribution to macrofungi of western part of Turkey, JFS, 27 (2004) 17-38.

22. Y. Uzun, A. Kaya, A. Keleş, M.E. Akçay, i. Acar, Macromycetes of Genc district (Bingöl-Turkey), Int. J. Bot., 5 (2009) 301-06.

23. S. Baydar, E. Sesli, Trabzon ili Akçaabat yöresinde belirlenen makromantarlar, Turk. J. Bot., 18 (1994) 99-101.

24. E. Sesli, i. Türkekul, Three new records for the Turkish mycoflora, Turk. J. Bot., 24 (2000) 259-62.

25. A. Kaya, Contributions to the macrofungi flora of Bitlis province, Turk. J. Bot., 25 (2001) 379-83.

26. I. Akata, B. Çetin, M. Işıloğlu, Macrofungal diversity of Ilgaz mountain national park and its environs (Turkey), Mycotaxon, 113 (2010) 287-90.

27. M.H. Solak, H. Allı, M. Işıloğlu, Macrofungi of Osmaniye province, Mantar Dergisi, 2 (2011) 1-7.

28. H. Allı, M. Işıloğlu, M.H. Solak, Macrofungi of Aydın Province, Turkey, Mycotaxon, 99 (2007) 163-65.

29. A. Afyon, D. Yağız, A. Türkoğlu, Sinanpaşa (Afyonkarahisar) yöresinin makromantarları üzerine bir çalışma, 21. Ulusal Biyoloji Kongresi, İzmir, (2012) $1165 p$.

30. H. Güngör, M.H. Solak, H. Allı, M. Işıloğlu, E. Kalmış, New macrofungi records to the Turkish mycobiota, BioDiCon, 7 (2014), 126-29.

31. Y. Uzun, I. Acar, M. E. Akçay, I. Akata, Additions to the Turkish Discomycetes, Turk. J. Bot., 38 (2014) 617-22.

32. H.H. Doğan, C. Öztürk, G. Kaşık, Two new Peziza records from Mut environ, The Herb J. System. Botan. 9 (2002) 117-22.

33. C. Öztürk, G. Kaşık, H.H. Doğan, S. Aktaş, Macrofungi of Alanya district, Turk. J. Bot., 27 (2003) 303-12.

34. H. Güngör, Ö.F. Çolak, M. Yaratanakul Güngör, M.H. Solak, New ascomycete (Geoglossum umbratile, Peziza lobulata) records for Turkey, BioDiCon, 8 (2015) 1-3.

35. F. Yilmaz Ersel, M.H. Solak, Three new records for the Turkish macromycota, Ekoloji, 13 (2004), 7-8.

36. H.H. Doğan, A. Türkoğlu, Macrofungal diversity of Hasandağı Mountain and Göreme District in Turkey, Mycologia Balcanica, 3 (2006) 173-78.

37. F. Gücin, Elazığ yöresinde yenebilen doğa mantarları ve yurdumuz makromantar florası için yeni kayıt olanlar, Türkiye II. Yemeklik Mantar Kongresi, Yalova Atatürk Bahçe Kültürleri Araştırma Enstitüsü, Yalova, (1984) 1-13.

38. F. Gücin, Elazığ çevresinde belirlenen makrofunguslar, Doğa-Tr. J. of Botany, 14 (1990) 171-77.
39. A. Öztürk, K. Demirel, İ.H. Arık, İnegöl (Bursa) çevresinde yetişen zehirli ve yenen mantarlar üzerinde sistematik, morfolojik ve ekolojik incelemeler, Fen Bil. Dergisi, 1 (1990) 27-38

40. M. Işıloğlu, R. Watling, Macromycetes of Mediterranean Turkey, EJB, 49 (1992) 99-121.

41. i. Acar, Y. Uzun, K. Demirel, A. Keleş, Macrofungal diversity of Hani (Diyarbakır/Turkey) district, BioDiCon, 8 (2015), 28-34.

42. Ö.F. Çolak, İ. Şen, N. Alkan, M. Işıloğlu, A new and interesting Peziza record from Sweet Gum forest in Turkey, Mantar Dergisi, 6 (2015) 10-12.

43. K. Demirel, Y. Uzun, A. Kaya, Macrofungi of Ağr province, Turk. J. Bot., 26 (2002) 291-95.

44. I. Akata, A. Kaya, Two new additions to Turkish Ascomycota, Int. J. Bot., 8 (2012) 79-81.

45. Y. Uzun, S. Kesici, i. Acar, Türkiye Pezizales'leri için Yüksekova (Hakkari) yöresinden iki yeni kayıt, I. Ulusal Mikoloji Günleri, Erzurum, (2014) 35p.

46. E. Sesli, Four interesting records of Pezizales of the macrofungal flora of Turkey, Turk. J. Bot., 22 (1998) 289-93.

47. C. Öztürk, HH. Doğan, G. Kaşık, Ermenek (Karaman) Makrofungus Florasına Illaveler, S.Ü. Fen-Edebiyat Fak. Fen Dergisi, 18 (2001) 61-66.

48. M. Işıloğlu, N. Öder, Contributions to the macrofung of Mediterranean Turkey, Turk. J. Bot., 19 (1995) 603 09.

49. A. Afyon, Macrofungi of Seydişehir District (Konya), Turk. J. Bot., 21 (1997), 173-76.

50. K. Gezer, Contributions to the macrofungi flora of Antalya Province, Turk. J. Bot., 24 (2000) 293-98.

51. D. Yağız, A. Afyon, M. Konuk, The macrofungi of Karabük province, Turk. J. Bot., 29 (2005) 345-53.

52. A. Türkoğlu, A. Kanlık, K. Gezer, Macrofungi of Çameli district (Denizli-Turkey), Turk. J. Bot., 31 (2007) 551-57.

53. H. Köstekci, M. Yamaç, M.H. Solak, Macrofungi of Türkmenbaba Mountain (Eskişehir), Turk. J. Bot., 29 (2005) 409-16.

54. A. Kaya, Macrofungal diversity of Nemrut Mount National Park and its environs (Adiyaman-Turkey), Afr. J. Biotechnol., 8 (2009) 2978-83.

55. M.H. Solak, H. Allı, M. Işıloğlu, H. Güngör, E. Kalmış, Contributions to the macrofungal diversity of Antalya province, Turk. J. Bot., 38 (2014) 386-97.

56. K. Demirel, Two new records for the mycoflora of Turkey, Turk. J. Bot., 21 (1997) 103-05.

57. K. Gezer, F. Taşkın Ekici, A. Türkoğlu, Macrofungi of Karcl Mountain (Denizli, Turkey), Turk. J. Bot., 32 (2008) 91-96.

58. M.H. Solak, M. Işıloğlu, E. Kalmış, H. Allı, Macrofungi of Turkey, Checklist (Vol. 1), Üniversiteliler Ofset, İzmir (2007) 254p.

59. J. Breitenbach, F. Kränzlin, Fungi of Switzerland, Ascomycetes (Vol. 1), Verlag Mycologia, Switzerland, (1984) 310p.

60. G.S. Barseghyan, S.P. Wasser, The genus Peziza Dill. ex Fr. (Pezizales, Ascomycota) in Israel, Ascomycete. org, 2 (2011) 39-50. 
